\title{
Plotgardener: Cultivating precise multi-panel figures in $\mathbf{R}$
}

\author{
Nicole E Kramer ${ }^{1}$, Eric S Davis ${ }^{1}$, Craig D Wenger², Erika M Deoudes ${ }^{3}$, \\ Sarah M Parker ${ }^{1}$, Michael I Love ${ }^{4,5}$, Douglas H Phanstiel ${ }^{1,3,6,7,8}$
}

\author{
Availability \\ Package: https://bioconductor.org/packages/plotgardener \\ Code: https://github.com/PhanstielLab/plotgardener \\ Documentation: https://phanstiellab.github.io/plotgardener/
}

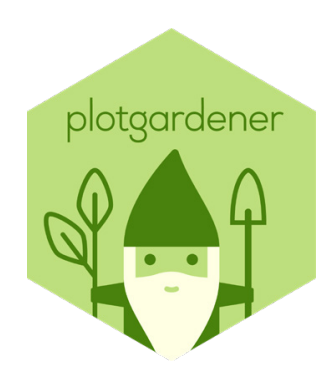

The $\mathrm{R}$ programming language is one of the most widely used programming languages for transforming raw genomic data sets into meaningful biological conclusions through analysis and visualization, which has been largely facilitated by infrastructure and tools developed by the Bioconductor project. However, existing plotting packages rely on relative positioning and sizing of plots, which is often sufficient for exploratory analysis but is poorly suited for the creation of publication-quality multi-panel images inherent to scientific manuscript preparation. We present plotgardener, a coordinate-based genomic data visualization package that offers a new paradigm for multi-plot figure generation in R. Plotgardener allows precise, programmatic control over the placement, aesthetics, and arrangements of plots while maximizing user experience through fast and memory-efficient data access, support for a wide variety of data and file types, and tight integration with the Bioconductor environment. Plotgardener also allows precise placement and sizing of ggplot 2 plots, making it an invaluable tool for $\mathrm{R}$ users and data scientists from virtually any discipline.

\section{Rationale}

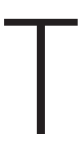
he increasing size, complexity, and sheer volume of multi-omic data sets has created a dire need for tools to efficiently visualize, interpret, and communicate the underlying biological signals present in these data. Towards this end, genome browsers, including the UCSC Genome Browser and IGV, have revolutionized our ability to investigate genomic data in a rapid and intuitive fashion, using a stacked linear representation of a wide variety of data types and annotations ${ }^{1-8}$. Recently, more specialized browsers like Juicebox ${ }^{9}$ and HiGlass ${ }^{10}$ have increased the ability to visualize non-linear data types, such as 3D chromatin contact frequency ${ }^{11,12}$. Furthermore, an ever-increasing array of programmatic libraries and browser APIs now allow code-based, integrated data analysis and construction of browser tracks, which has improved reproducibility and automation ${ }^{13-17}$.

While these tools have been transformative for data exploration, they are largely based on single-panel figures and vertical stacking of genomic tracks and are often ill-suited for the generation of complex multi-panel figures that include both genomic and non-genomic plot types. Such complex figures are often critical for evalu- ating the underlying biology and are almost always used to present multi-omic data in publications. Thus, a tool specifically designed to programmatically create and arrange publication-quality multi-panel figures is critical to extend the rigor, reproducibility, and clarity of scientific data visualizations.

Currently existing $\mathrm{R}$ packages like patchwork ${ }^{18}$, cowplot $^{19}$, gridExtra ${ }^{20}$ and Sushi ${ }^{21}$ (which was developed by our group) can be used to arrange multi-panel plots. However, these layout packages use relative positioning to place plots, giving users little control over precise sizing and arrangement. Figures generated with these tools often need finishing in graphic design software such as Adobe Illustrator ${ }^{22}$, Inkscape ${ }^{23}$, PowerPoint ${ }^{24}$, and Keynote $^{25}$. In addition to the cost of purchasing proprietary graphic design software and the steep learning curve often associated with their use, generating multi-panel figures with these software requires non programmatic, manual user interactions, a labor intensive process that decreases reproducibility.

Here we introduce plotgardener, an $\mathrm{R}$ package for absolute coordinate-based plot placement and sizing of

'Curriculum in Bioinformatics and Computational Biology, University of North Carolina at Chapel Hill, Chapel Hill, NC, USA. ${ }^{2}$ Independent Scholar, Issaquah, WA, USA. ${ }^{3}$ Thurston Arthritis Research Center, University of North Carolina at Chapel Hill, Chapel Hill, NC, USA. ${ }^{4}$ Department of Biostatistics, University of North Carolina at Chapel Hill, Chapel Hill, NC, USA. ${ }^{5}$ Department of Genetics, University of North Carolina at Chapel Hill, Chapel Hill, NC, USA. ${ }^{6}$ Department of Cell Blology \& Physiology, University of North Carolina at Chapel Hill, Chapel Hill, NC, USA. ZLineberger Comprehensive Cancer Center, University of North Carolina at Chapel Hill, Chapel Hill, NC, USA. ${ }^{8}$ Curriculum in Genetics \& Molecular Biology, University of North Carolina at Chapel Hill, Chapel Hill, NC, USA.

Contact: douglas_phanstiel@med.unc.edu 
$\mathbf{a}$

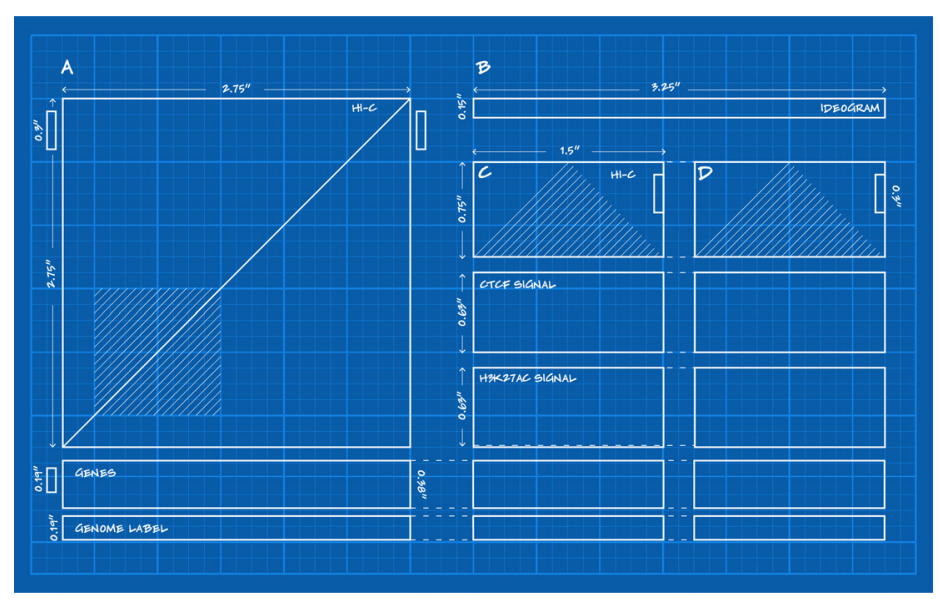

b

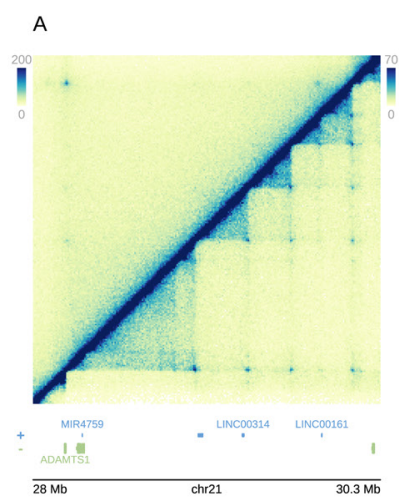

B

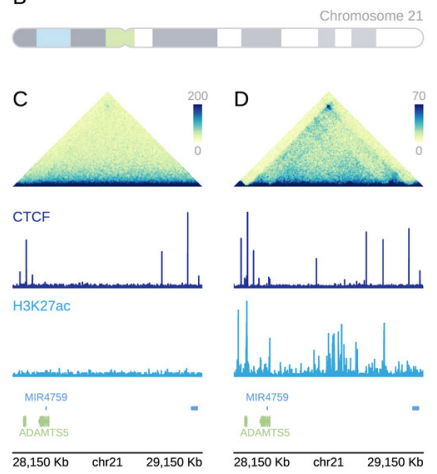

Fig. 1 | Plotgardener uses a coordinate-based plotting system to size and arrange plots. a, Blueprint outline of a multi-omic figure to be created with specified dimensions and placements on a defined page. b, Multipanel, multi-omic figure programmatically created with plotgardener using the sizing and placement coordinates from (a). The plotgardener functions used to create this figure include pageCreate, plotHicSquare, annoHeatmapLegend, plotGenes, annoGenomeLabel, plotldeogram, plotHicTriangle, plotSignal, and plotText. Code to reproduce this plot is included in the plotgardener package.

complex multi-panel plots. This paradigm gives users precise control over size, placement, typefaces, font sizes, and virtually all plot aesthetics without the need for graphic design software. Plotgardener (1) supports a vast array of genomic data types, (2) allows precise placement and sizing of genomic and non-genomic figures, (3) is tightly integrated with the Bioconductor environment ${ }^{26}$, and (4) is optimized for speed and user-experience. The code is open source, extensively documented, and freely available via GitHub and Bioconductor.

\section{Philosophy}

The defining feature of plotgardener that separates it from virtually all other genomic visualization tools is that it allows exact sizing and placement of plots using an absolute, coordinate-based plotting system (Fig. 1). Each plot, axis, and annotation is placed independently according to user-specified positions and dimensions. Each plot or feature extends from edge to edge of the defined coordinates, allowing for precise control and perfect alignment of plots. Rulers and guidelines can be temporarily added for ease of plotting and then removed prior to file generation. Adding additional plots does not shift or resize existing ones, so figures can be built incrementally and adjusted without affecting other figure panels, allowing rapid and easy construction of publication-quality multi-panel figures.

\section{Data types}

Plotgardener can display a vast array of genomic data types which can be provided as either external files or $\mathrm{R}$ data classes. Plotgardener has 45 functions for plotting and annotating diverse genomic data types, including genome sequences, gene/transcript annotations, chromo- some ideograms, signal tracks, GWAS Manhattan plots, genomic ranges (e.g. peaks, reads, contact domains, etc), paired ranges (e.g. paired-end reads, chromatin loops, structural rearrangements, QTLs, etc), and 3D chromatin contact frequencies. Plotgardener automatically recognizes and reads compressed, indexed file types including “.bam”, “.bigwig”, and “.hic”, allowing for rapid and memory-efficient reading and plotting of large genomic data. Fig. 2 displays the runtime required to read and plot various types of genomic data. Even with file sizes exceeding $50 \mathrm{GBs}$, plotgardener can read and plot data in under a second. Multiple classes of $\mathrm{R}$ objects are supported, including "data.frame”, "data.table”, "tibble”, "GRanges”, and "GInteractions". Plotgardener automatically detects whether the input is a file path or an R object and handles them accordingly, providing a seamless and flexible experience for the user.

\section{Bioconductor integration}

Plotgardener is tightly integrated with the Bioconductor ecosystem ${ }^{26}$, making it compatible with many existing workflows. It has 29 built-in genomes and associated annotations but can easily accommodate custom genomes and annotations using Bioconductor $\mathrm{TxDb}^{27}, \mathrm{OrgDb}^{28}$, and BSgenome ${ }^{29}$ packages and/or objects. Plotgardener leverages these annotation resources on behalf of the user to obtain and plot chromosome sizes, gene and transcript structures, and nucleotide sequences. By preconfiguring the genome builds and associated feature data, plotgardener allows users to focus their attention on layout and to quickly visualize their data rather than spending time and effort on curation and organization of sequences and genome annotations. 


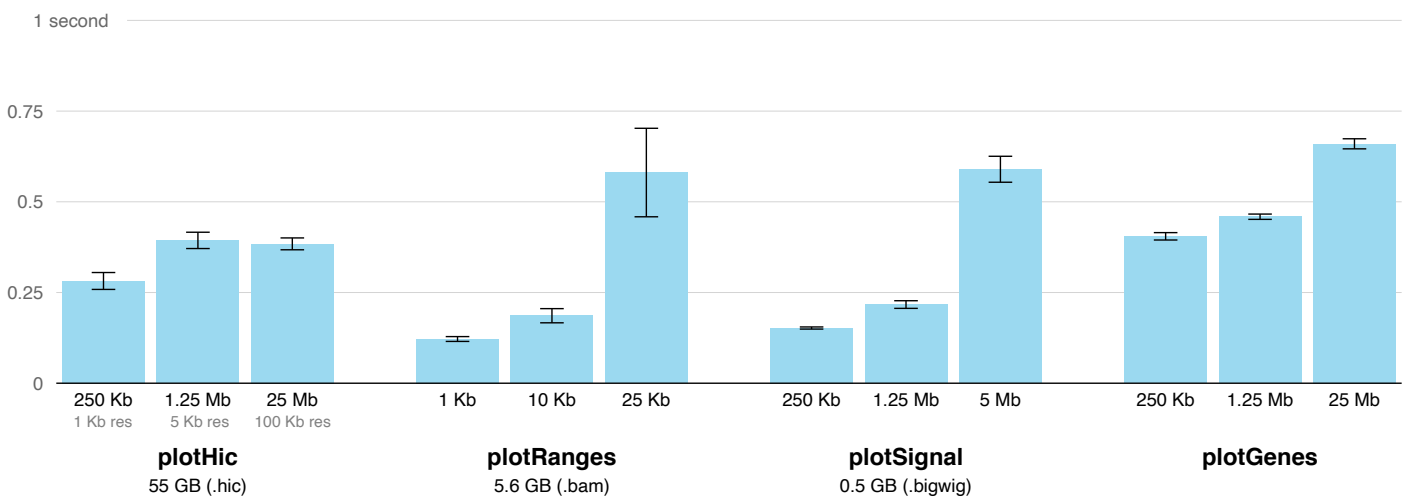

Fig. 2 | Plotgardener function runtimes. A bar plot depicting mean runtimes for reading and plotting genomic data across various sizes and resolutions using plotHicSquare, plotRanges, plotSignal, and plotGenes functions. Times were calculated for 20 randomly chosen gene regions for each bar. Error bars indicate standard error. File sizes for the input data are indicated below each set of bars for each function: plotHicSquare (55 GB .hic file), plotRanges (5.6 GB .bam file), plotSignal (0.5 GB .bigwig), plotGenes (NA, data stored as an internal object).

\section{User experience}

Plotgardener includes a variety of user-friendly features to maximize ease of use for both novices and experienced $\mathrm{R}$ programmers. We describe just some of these features here. Parameters can be set within each function call or passed in a pgParams object for more efficient code. Genomic coordinates can be set either by supplying the chromosome, start, and end position or by providing a gene name (e.g. IL1B), reference genome name (e.g. "hg19"), and optional base pair window around the gene (e.g. 50,000 bp). Resolution of Hi-C contact matrices, signal tracks, and gene tracks are automatically determined based on the genomic range being plotted, but can be overwritten if desired. When genomic regions are too large to label all genes, plotGenes and plotTranscripts will choose which genes/transcripts to label based on frequency of appearance in publications. Users can provide their own priorities or select individual genes to highlight with text and colors. A "colorby" function allows users to flexibly color genomic features by quantitative and qualitative attributes. Plotgardener is open source, version controlled, and extensively documented via articles and vignettes (https://phanstiellab.github.io/plotgardener/).

\section{ggplot and beyond}

In addition to its included functions for plotting and annotating genomic data, plotgardener allows for the absolute sizing and placement of non-genomic plots within a plotgardener page. Users can make multi-panel figures seamlessly by integrating and aligning plotgardener and non-plotgardener plots or create coordinate-based layouts entirely composed of external plot types and objects. For example, plotgardener was used to arrange and add text annotations to the ggplot2 plot objects featured in Fig. 3. Plotgardener intuitively sizes, arranges, and overlays plots, text, and geometric objects to make complex figure arrangements beyond basic grid-style or relative layouts. We are actively developing the package and potential future additions include more plotting functions, templates for common arrangements, convenient functions for multiplotting, enhanced ggplot2 integration, and more.

In summary, plotgardener provides a new paradigm for generating complex publication-quality figures of both genomic and non-genomic data types, making it an invaluable tool for $\mathrm{R}$ users and data scientists from virtually any discipline.

\section{Acknowledgments}

We would like to thank Hyejung Won and Jason Stein for helpful discussions and feedback. We thank Muhammad Saad Shamim and Neva Durand for assistance with the strawr package. This work was supported by NIH grants (R35-GM128645 to D.H.P.). N.E.K. and E.S.D. were supported by the NIH-NIGMS training grant T32-GM067553. S.M.P. is supported by the NSF GRFP DGE-1650116. M.I.L. was supported by NIH grants R01-MH118349 and R01HG009937.

\section{Author information}

Nicole E Kramer: Conceptualization, Software, Methodology, Writing

Eric S Davis: Conceptualization, Software, Writing

Craig D Wenger: Software

Erika M Deoudes: Visualization

Sarah M Parker: Software

Michael I Love: Software, Writing

Douglas H Phanstiel: Conceptualization, Writing, Supervision, Funding acquisition

\section{Methods}

Visualization methods. Plotgardener is an open-source extension for $\mathrm{R}$, building its visualization functions from primitive graphical functions in the grid package ${ }^{30}$. Each plot and annotation is drawn within its own defined graphical region, or viewport, and then placed on a larger 

available under aCC-BY-NC-ND 4.0 International license.

Thousands of COVID-19 Cases per 100,000 People

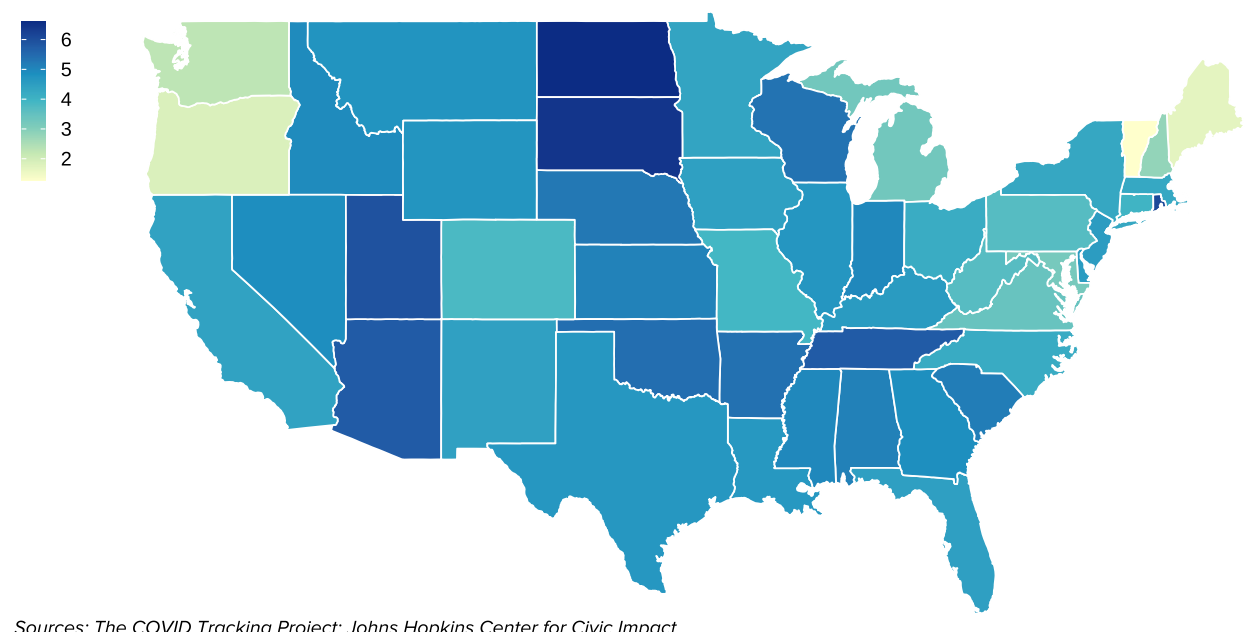

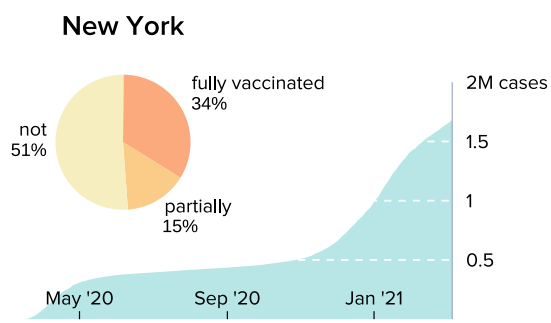

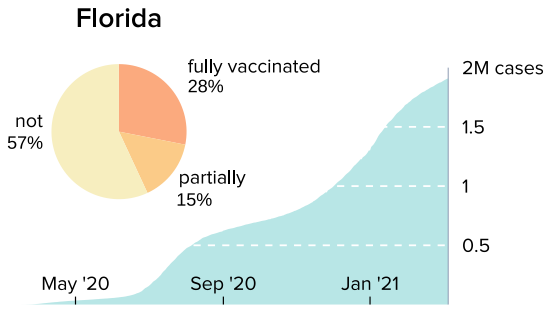

Fig. 3 | Precise arrangement of ggplot2 objects with plotgardener. Five ggplot2 objects and additional text elements were arranged into this multi-panel figure using plotgardener. Left: A map of the United States depicts COVID-19 cases per 100,000 people in each state. Right: Pie charts depict state vaccination percentages and line plots describe cumulative COVID-19 cases in New York and Florida. The plotgardener functions used to create this figure include pageCreate, plotGG, and plotText. Code to reproduce this plot is included in the plotgardener package.

plotgardener page. These viewports give the power to specify the size and placement of plot containers and clip data to precise genomic and data axis measurements. To obtain large, reference genomic annotation data, plotgardener integrates and utilizes packages and data objects through Bioconductor.

Gene and transcript label publication frequency mining. Annotations for genes in PubMed articles were obtained from the PubTator text mining tool ${ }^{31}$ and counted for each unique gene ID. Publication frequencies were matched via gene ID to Bioconductor transcript database (TxDb) gene IDs for the 29 built-in plotgardener genomes.

Evaluating runtimes of plotgardener plotting functions. To calculate plotgardener plotting runtimes, we used the $\mathrm{R}$ package microbenchmark ${ }^{32}$. plotHicSquare, plotSignal, plotGenes, and plotRanges functions were timed for various genomic region sizes and resolutions. Each condition was timed on 20 random genomic regions generated by BedtoolsR ${ }^{33}$.

\section{Data availability}

Various publicly available datasets are included with a supplementary plotgardenerData package and were used to demonstrate the functionalities of plotgardener. Hi-C datasets from the GM12878 and IMR90 cell lines were downloaded from $\mathrm{GEO}^{34}$ under the accession code GSE63525. CTCF ChIP-seq signal files for the GM12878 and IMR90 cell lines were downloaded from the ENCODE portal ${ }^{35}$ with accession codes ENCFF312KXX and ENCFF603PYX. H3K27ac ChIP-seq signal files for the GM12878 and IMR90 cell lines were downloaded from the NIH Roadmap Epigenomics Project ${ }^{36}$ with reference epigenome identifiers E116 and E017. COVID-19 case data was downloaded from The COVID Tracking Project (https://covidtracking.com/). State population data and state COVID-19 vaccination data were downloaded from the Johns Hopkins Centers for Civic Impact COVID-19 GitHub repository (https://github.com/govex/COVID-19/).

\section{References}

1. Kent, W. J. et al. The human genome browser at UCSC. Genome Res. 12, 996-1006 (2002).

2. Thorvaldsdóttir, H., Robinson, J. T. \& Mesirov, J. P. Integrative Genomics Viewer (IGV): high-performance genomics data visualization and exploration. Brief. Bioinform. 14, 178-192 (2013).

3. Zhou, X. et al. The Human Epigenome Browser at Washington University. Nat. Methods 8, 989-990 (2011).

4. Freese, N. H., Norris, D. C. \& Loraine, A. E. Integrated genome browser: visual analytics platform for genomics. Bioinformatics 32, 2089-2095 (2016).

5. Flicek, P. et al. Ensembl 2011. Nucleic Acids Res. 39, D800-6 (2011).

6. Abeel, T., Van Parys, T., Saeys, Y., Galagan, J. \& Van de Peer, Y. GenomeView: a next-generation genome browser. Nucleic Acids Res. 40, e12 (2012).

7. Chelaru, F., Smith, L., Goldstein, N. \& Bravo, H. C. Epiviz: interactive visual analytics for functional genomics data. Nat. Methods 11, 938-940 (2014).

8. Carver, T., Thomson, N., Bleasby, A., Berriman, M. \& Parkhill, J. DNAPlotter: circular and linear interactive genome visualization. Bioinformatics 25, 119-120 (2009). 
9. Durand, N. C. et al. Juicebox Provides a Visualization System for Hi-C Contact Maps with Unlimited Zoom. Cell Syst 3, 99-101 (2016).

10. Kerpedjiev, P. et al. HiGlass: web-based visual exploration and analysis of genome interaction maps. Genome Biol. 19, 125 (2018).

11. Wang, Y. et al. The 3D Genome Browser: a web-based browser for visualizing 3D genome organization and long-range chromatin interactions. Genome Biol. 19, 151 (2018).

12. Djekidel, M. N., Wang, M., Zhang, M. Q. \& Gao, J. HiC-3DViewer: a new tool to visualize Hi-C data in 3D space. Quantitative Biology 5, 183-190 (2017).

13. Yin, T., Cook, D. \& Lawrence, M. ggbio: an R package for extending the grammar of graphics for genomic data. Genome Biol. 13, R77 (2012).

14. Hahne, F. \& Ivanek, R. Visualizing Genomic Data Using Gviz and Bioconductor. in Statistical Genomics: Methods and Protocols (eds. Mathé, E. \& Davis, S.) 335-351 (Springer New York, 2016).

15. Lawrence, M., Gentleman, R. \& Carey, V. rtracklayer: an R package for interfacing with genome browsers. Bioinformatics 25, 1841-1842 (2009).

16. Wickham, H. ggplot2: Elegant Graphics for Data Analysis. (2016).

17. Durinck, S., Bullard, J., Spellman, P. T. \& Dudoit, S. GenomeGraphs: integrated genomic data visualization with R. BMC Bioinformatics 10, 2 (2009).

18. Pedersen, T. L. patchwork: The Composer of Plots. (2020).

19. Wilke, C. O. cowplot: Streamlined Plot Theme and Plot Annotations for 'ggplot2'. (2020).

20. Auguie, B. gridExtra: Miscellaneous Functions for 'Grid' Graphics. (2017).

21. Phanstiel, D. H., Boyle, A. P., Araya, C. L. \& Snyder, M. P. Sushi.R: flexible, quantitative and integrative genomic visualizations for publication-quality multi-panel figures. Bioinformatics 30, 2808-2810 (2014).

22. Adobe Inc. Adobe Illustrator. (2019).

23. Inkscape Project. Inkscape. (2020).

24. Microsoft Corporation. Microsoft PowerPoint. (2018).

25. Apple Inc. Keynote.

26. Gentleman, R. C. et al. Bioconductor: open software development for computational biology and bioinformatics. Genome Biol. 5, R80 (2004).

27. Lawrence, M. et al. Software for Computing and Annotating Genomic Ranges. PLoS Computational Biology vol. 9 (2013).

28. Pagès, H., Carlson, M., Falcon, S. \& Li, N. AnnotationDbi: Manipulation of SQLite-based annotations in Bioconductor. (2021).

29. Pagès, H. BSgenome: Software infrastructure for efficient representation of full genomes and their SNPs.
(2021).

30. R Core Team. R: A Language and Environment for Statistical Computing. (2021).

31. Wei, C.-H., Kao, H.-Y. \& Lu, Z. PubTator: a web-based text mining tool for assisting biocuration. Nucleic Acids Res. 41, W518-22 (2013).

32. Mersmann, O. microbenchmark: Accurate Timing Functions. (2019).

33. Patwardhan, M. N., Wenger, C. D., Davis, E. S. \& Phanstiel, D. H. Bedtoolsr: An R package for genomic data analysis and manipulation. J Open Source Softw 4, (2019).

34. Barrett, T. et al. NCBI GEO: archive for functional genomics data sets--update. Nucleic Acids Res. 41, D991-5 (2013).

35. ENCODE Project Consortium. An integrated encyclopedia of DNA elements in the human genome. Nature 489, 57-74 (2012).

36. Bernstein, B. E. et al. The NIH Roadmap Epigenomics Mapping Consortium. Nat. Biotechnol. 28, 1045-1048 (2010). 